\title{
Características Morfológicas y Biométricas del Músculo Papilar Septal en Corazones de Individuos Chilenos
}

\author{
Morphological and Biometric Characteristics of \\ Septal Papillary Muscle in Hearts of Chilean Individuals
}

*Aglae Soto Peralta \& ** Jorge Henríquez Pino

SOTO, A. \& HENRÍQUEZ, J. Características morfológicas y biométricas del músculo papilar septal en corazones de individuos Chilenos. Int. J. Morphol., 29(3):711-715, 2011.

RESUMEN: Se realizó un estudio descriptivo de las características anatómicas y biométricas del músculo papilar septal en 30 corazones de individuos adultos chilenos, en edades comprendidas entre 18 y 84 años de edad, de ambos sexos, sin aparente patología cardíaca, pertenecientes a la Unidad de Anatomía Humana Normal del Departamento de Ciencias Básicas de la Universidad de La Frontera. Los resultados mostraron que el músculo papilar septal, se presenta en un 83,3\% de los corazones del estudio. De éstos, el $44,0 \%$ presenta un solo músculo, el $28 \%$ presenta dos músculos y el $28 \%$ tres músculos papilares septales. De los músculos papilares septales encontrados, el $71,1 \%$ correspondió a la forma cono libre, el $24,4 \%$ a la forma cono pegado y el 4,5\% a la forma puente. En cuanto a la longitud de forma cono pegado y cono libre, el rango que se encuentra en mayor porcentaje está entre 4,0 mm-5,99 mm; con un $45,5 \%$ y $42,4 \%$, respectivamente. Este estudio nos demuestra la importancia de incorporar al músculo papilar septal a la nomenclatura anatómica internacional.

PALABRAS CLAVE: Anatomía; Corazón; Ventrículo derecho; Músculo papilar septal.

\section{INTRODUCCIÓN}

El corazón de los vertebrados primitivos es un tubo vascular modificado constituido por compartimientos alineados en serie. En sentido céfalo-caudal se encuentra el seno venoso, el atrio primitivo, el ventrículo primitivo, entre las cámaras primitivas hay dispositivos valvulares rudimentarios. En los mamíferos el corazón es tetracameral, en los que la tabicación ventricular es completa (García-Porrero \& Hurlé, 2005).

Un estudio de Skwarek et al. (2006), refiere que la hipótesis de Szostakiewicz señala que los músculos papilares emigraron durante la evolución a lo largo de las paredes del ventrículo derecho a medida que la valva tricúspide cambió desde una forma de media luna hacia la forma actual. Los tipos de conexiones entre la valva tricúspide y los músculos papilares han cambiado durante la filogénesis desde conexiones directas y membranosas a cuerdas tendinosas, como se refleja en la ontogénesis.
La valva atrioventricular derecha, consiste en pliegue de endocardio, reforzado por tejido fibroso, presenta tres cúspides: anterior, posterior y septal, que reciben a las cuerdas tendinosas que son cordones fibrosos, cuyos extremos se insertan en los músculos papilares y/o pared ventricular que presenta las trabéculas carnosas que pueden ser crestas, puentes o músculos papilares que sobresalen de la pared.

Los músculos papilares del ventrículo derecho son: uno anterior, grande, cuyas cuerdas tendinosas se dirigen a la cúspide anterior y posterior de la valva atrioventricular; uno posterior, más pequeño e irregular, presentando a veces dos o más segmentos, desde él las cuerdas tendinosas pasan a las cúspides posterior y septal de la valva atrioventricular; y músculo papilar septal pequeño, pudiendo ser más de uno, de forma variable e inconstante, sus cuerdas tendinosas se fijan a la cúspide anterior y septal de la valva atrioventricular. 
Existen autores clásicos que han descrito el músculo papilar septal, tales como Testut \& Latarjet (1972), que se refiere al músculo como pequeños mamelones carnosos que ocupa la parte inferior del infundíbulo, llamándolo músculo papilar del cono arterial, también llamado como el "músculo de Lancisi", de una longitud de $6-8 \mathrm{~mm}$. Bouchet \& Cuilleret (1979) refieren al músculo papilar septal como el músculo papilar del cono arterioso de Luschka. Gardner et al. (1971) hablan de pequeños grupos de músculos septales cónicos, de tamaño y forma variable, de los cuales uno puede ser mayor y más constante.

Como podemos observar existe controversia en relación a la presencia y al número de músculos papilares en ventrículo derecho. Por tal motivo surge el interés de conocer las características anatómicas y biométricas del músculo papilar septal en corazones humanos de individuos Chilenos.

\section{MATERIAL Y MÉTODO}

Se realiza un estudio descriptivo y transversal de características anatómicas y biométricas del músculo papilar septal en corazones de individuos adultos Chilenos, cuyo universo está constituido por 30 corazones de individuos adultos chilenos, pertenecientes a la Unidad de Anatomía Humana del Departamento de Ciencias Básicas de la Universidad de La Frontera, con edades comprendidas entre 18 y 84 años de edad, de ambos sexos, siendo 22 del sexo masculino y 8 del sexo femenino y sin aparente patología cardiaca.

La retirada de los corazones fue ejecutada en los procesos de disección realizados en los cadáveres. Los corazones obtenidos fueron identificados mediante el uso de placas plásticas numeradas y en un registro propio se anotaban los datos correspondientes.

Los corazones fueron fijados en una solución de formalina al $10 \%$, previo lavado con agua corriente por un período aproximado de dos horas.

Se sacaron los corazones del recipiente con formalina y se lavaron con agua corriente, retirando el exceso de formol de las cavidades de cada corazón.

Se procedió a la disección de cada corazón realizando un corte diagonal en la pared lateral ventricular derecha, desde el margen del anillo atrioventricular derecho hasta su ápice, usando bisturí y tijeras dejando al descubierto la pared septal del ventrículo.
Se realizó una observación directa a cada ventrículo derecho con una lupa de aumento de 3 veces, para registrar detalles macroscópicos de la pared septal.

Para efectuar las fotografías se montaron los corazones en un atril en un plano vertical para quedar con su pared septal descubierta y fija, luego se procedió a la toma fotográfica, usando una cámara marca Nikon Coolpix 3.2, con zoom, de la pared septal de cada uno de los corazones.

Posteriormente, se realizó medición de las fotografías con el método Carnoy para ser registradas en el programa de computación Excel.

La clasificación que se realizó según forma del músculo papilar septal fue la siguiente:

Cono libre: para aquel sobrelevantamiento muscular con forma de cono de base pegada a la pared ventricular y ápice libre donde nacen las cuerdas tendinosas para fijarse a las cúspides.

Cono pegado: aquel sobrelevantamiento muscular con forma de cono que no tiene ápice libre.

Puente: aquel sobrelevantamiento muscular en la pared de forma alargado.

Para el análisis de los datos se utilizaron los métodos y técnicas propias de los estudios descriptivos en el programa computacional Excel. Para establecer media, mediana, desviación estándar y chi-cuadrado (grado de significancia) se utilizó el programa computacional SPSS.

\section{RESULTADOS}

De acuerdo a las variables observadas encontramos los siguientes resultados:

De los 30 corazones del estudio, el músculo papilar septal se encontró presente en 25 de ellos, correspondiendo al $83,3 \%$ de los casos y hubo ausencia del músculo papilar septal en 5 corazones correspondiendo al $16,7 \%$ de los casos.

Respecto a la distribución de los corazones de acuerdo al número de músculos papilares septales presentes en pared septal del ventrículo derecho, nuestro estudio nos revela que en $11 / 25(44,0 \%)$ presentaron un solo músculo papilar septal; $7 / 25(28,0 \%)$ dos músculos papilares septales y $7 / 25(28,0 \%)$ tres músculos papilares septales. 


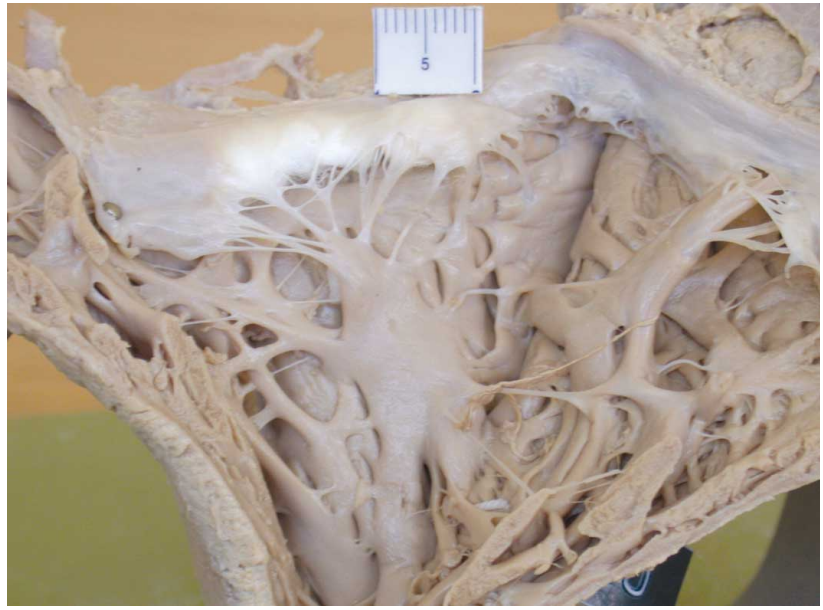

Fig. 1. Observación 20 (masculino, 54 años). Músculo papilar septal con forma de cono libre, cuerdas tendinosas insertadas en la cúspide anterior y septal de la válvula tricúspide.

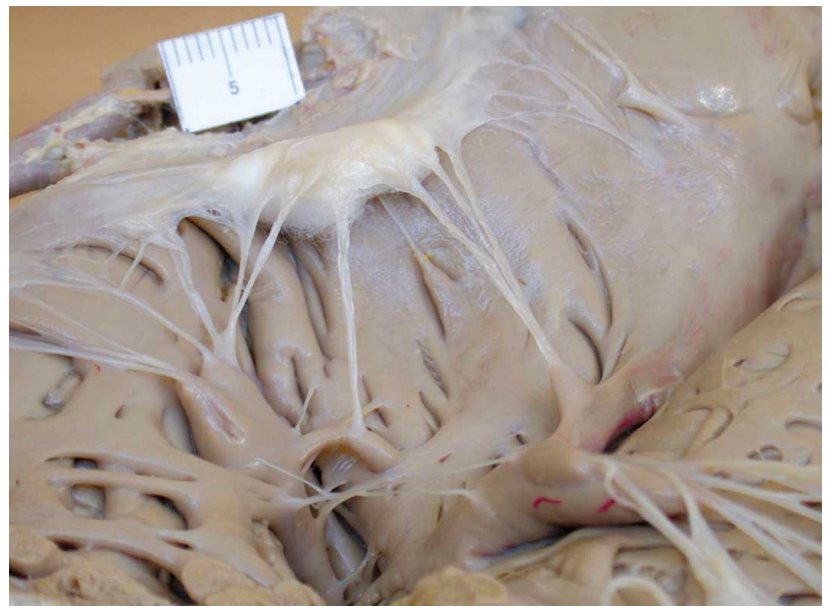

Fig. 3. Observación 21 (masculino 45 años). Muestra al músculo papilar septal con forma de puente, y su cuerda tendinosa insertándose en la cúspide septal de la válvula tricúspide.

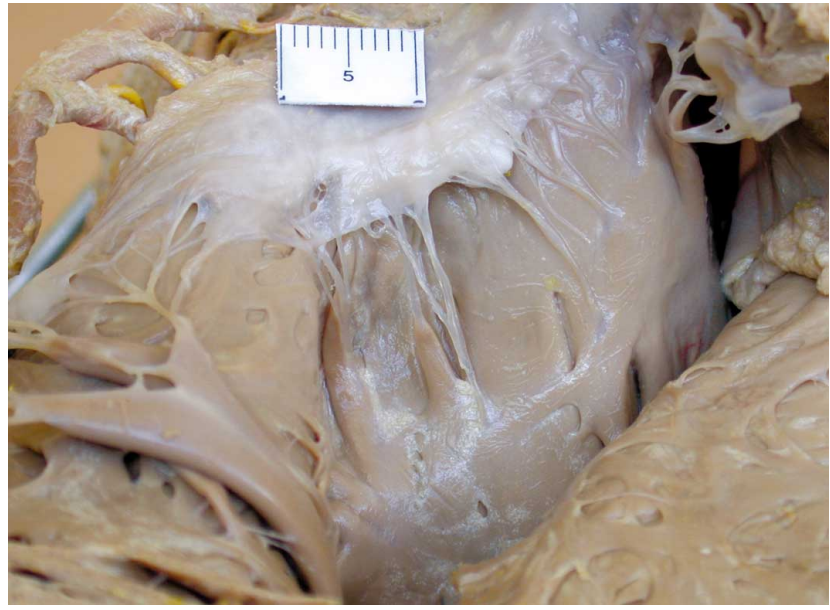

Fig. 2. Observación 14 (femenino 32 años). Se muestra el músculo papilar con forma de cono pegado, lateral a él, se observa una cuerda tendinosa directamente de la pared a la cúspide.

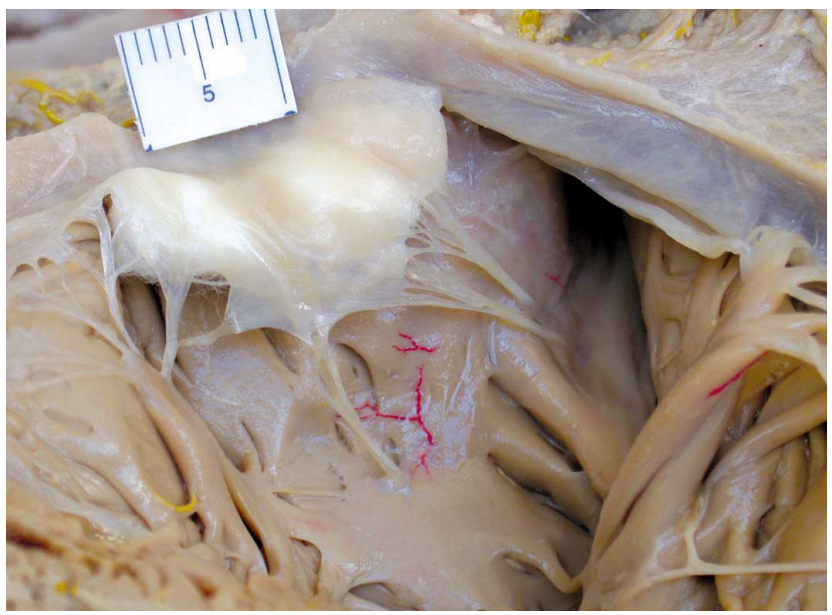

Fig. 4. Observación 28 (masculino 65 años). En la pared septal sólo hay presencia de cuerdas tendinosas.

Tabla I. Distribución de los corazones de acuerdo a la longitud del músculo papilar septal con forma de cono libre.

\begin{tabular}{lcc}
\hline Longitud del papilar septal forma de cono libre & Número & Porcentaje \\
\hline Entre $2,0-3,99 \mathrm{~mm}$ & 13 & 39,3 \\
Entre $4,0-5,99 \mathrm{~mm}$ & 14 & 42,4 \\
Entre $6,0-7,99 \mathrm{~mm}$ & 2 & 6,1 \\
Entre $8,0-9,99 \mathrm{~mm}$ & 2 & 6,1 \\
Entre 10 y más & 2 & 6,1 \\
Total & 33 & 100 \\
\hline
\end{tabular}

Tabla II. Distribución de los corazones de acuerdo a la longitud del músculo papilar septal con forma de cono pegado.

\begin{tabular}{lcc}
\hline Longitud del papilar septal forma de cono pegado & Número & Porcentaje \\
\hline Entre $2,0-3,99 \mathrm{~mm}$ & 3 & 27,3 \\
Entre $4,0-5,99 \mathrm{~mm}$ & 5 & 45,5 \\
Entre $6,0-7,99 \mathrm{~mm}$ & 2 & 18,1 \\
Entre $8,0-9,99 \mathrm{~mm}$ & 1 & 9,1 \\
Total & 11 & 100 \\
\hline
\end{tabular}

Al identificar la forma del músculo papilar septal, encontramos que de un total de 46 músculos papilares septales en los 25 corazones con presencia de éste, 33/46 (71,1\%) corresponden a cono libre (Fig. $1) ; 11 / 46(24,4 \%)$ corresponden a cono pegado (Fig. 2); y $2 / 46(4,5 \%)$ a forma de puente (Fig. 3). 


\section{DISCUSIÓN}

De los 30 corazones estudiados se encontró en un $83,3 \%$ presencia de músculo papilar septal y ausencia de este en el $16,7 \%$, lo que coincide con lo señalado por Nigri et al. (2001), en un $21,5 \%$ de ausencia. Esto demuestra que en la mayoría de los corazones existe este músculo, Luschka ya lo señalaba en el siglo XVII y lo describió como constante en la pared septal del ventrículo derecho denominándolo músculo del cono arterial, por lo cual Quiroz (1993) y Testut \& Latarjet lo describieron como músculo de Luschka o de Lancisi y de presencia constante.

Otros autores como Testut \& Latarjet, Williams \& Warwick (1985), Restivo et al. (1989), Quiroz, Rouviere \& Delmas (1999) y Drake et al. (2005) lo describieron como inconstante y cuando existe, puede ser uno o más de pequeños músculos papilares septales en la pared del ventrículo derecho, lo que también observamos en nuestro trabajo.

Fue descrito por Goss (1976) como un pequeño músculo llamado músculo papilar del infundíbulo ubicado en el extremo de la cresta supraventricular.

En tanto autores como Snell (2000), no describe la presencia de músculo papilar septal del ventrículo derecho, y sólo se refiere a los músculos papilares anterior y posterior, al igual que el Comité Internacional de Nomenclatura Anatómica. No compartimos esta postura ya que el músculo papilar septal lo observamos en el $83,3 \%$ de los casos de nuestro estudio.

El estudio morfológico realizado por Wenink (1977), del complejo papilar medial en 100 corazones embrionarios, fetales, neonatos y sujetos adultos sobre 60 años, observó presencia de músculo papilar septal con forma de cono y cuerdas tendinosas en todos los corazones neonatales e infantiles. En cambio en ancianos el músculo papilar septal real no se encontró, sólo se observaron cuerdas tendinosas insertadas en el septum, el autor afirma que el desarrollo cardiaco sigue después del nacimiento, tomando en consideración la hipertrofia y atrofia, y que termina con la muerte del individuo. Nuestro estudio fue realizado en personas adultas por lo que no podemos realizar comparaciones con lo señalado por Wenink.

La presencia del músculo papilar septal señalado por Nigri et al., es de un 78,5\% de los adultos, lo cual está de acuerdo con lo señalado en nuestra investigación.

Nuestra investigación nos reveló la presencia de un solo músculo papilar septal, en 44,0\% de los casos, lo que también fue observado por Nigri et. al., que señalaron un solo músculo en el 53,2\%. El músculo papilar septal con 2 cabezas lo observamos en un $28 \%$ de los casos, Nigri et al. lo observaron sólo en $16,5 \%$. El músculo papilar septal con 3 o más cabezas lo encontramos en $28 \%$ de los casos, Nigri et al. lo encontraron el $12,7 \%$.

Sin embargo, Bouchet \& Cuilleret lo describieron como pequeñas prominencias musculares que nacen desde la pared septal, pero no lo consideraron como músculo papilar septal propiamente tal, lo que no fue observado en nuestro trabajo.

La forma del músculo papilar septal la observamos como cono libre, cono pegado y puente; siendo el cono libre la de mayor porcentaje $(71,1 \%)$ y la forma de puente la de menor porcentaje $(4,5 \%)$. En la literatura revisada no se hallaron estudios sobre esta variable, sin embargo, Williams \& Warwick y Testut \& Latarjet describieron al músculo papilar septal como un cono de base pegada a la pared ventricular con vértice donde nacen las cuerdas tendinosas, o que correspondería a la forma de cono libre de nuestro estudio.

La longitud del músculo papilar septal ya sea como, cono pegado, cono libre o puente, observamos que en los tres, el porcentaje estuvo entre 4,0 - 5,99 mm, Nigri et al. registraron sólo la longitud del músculo papilar con una cabeza, que sería de 5,59 $\mathrm{mm}$ lo cual esta de acurdo a nuestros hallazgos. En cambio Testut \& Latarjet refiriéndose al músculo papilar del cono arterial, señalan una longitud de 6,0 a $8,0 \mathrm{~mm}$ mayor a la referidas en nuestro estudio. Para la forma de puente y cono pegado la literatura revisada no se encontraron referencias al respecto.

En conclusión, el músculo papilar septal está presente en un alto porcentaje $(83,3 \%)$. El número de estos músculos varían desde uno a tres, siendo más frecuente la presencia de un solo músculo papilar septal $(44,0 \%)$. El músculo papilar septal presenta forma de puente, cono libre y cono pegado, siendo la forma más frecuente la de cono libre $(71,1 \%)$. Finalmente, los rangos de longitud del músculo papilar septal, para la forma de cono pegado y cono libre varían entre 4,0 y 5,99 $\mathrm{mm}$.

SOTO, A. \& HENRÍQUEZ, J. Morphological and biometric characteristics of the septal papillary muscle in hearts of Chilean individuals. Int. J. Morphol., 29(3):711-715, 2011.

SUMMARY: A descriptive research study was carried out on the anatomical and biometric characteristics of the septal papillary muscle in 30 hearts of adult Chilean subjects, between 
18 and 84 years of age of both sexes and without apparent cardiac pathology, from the Normal Human Anatomy Unit, Basic Sciences Department of the Universidad de La Frontera. Results show that the septal papillary muscle was present in $83.3 \%$ of the hearts in the study. Of these $44.0 \%$ show one muscle only, $28 \%$ show two muscles and $28 \%$ show three septal papillary muscles. In the septal papillary muscles found, $71.1 \%$ are free cone-shaped, $24.4 \%$ were attached cone-shaped, and $4.5 \%$ were bridge-shaped. Regarding the length of attached cone and free cone shaped, the highest percentage range was between $4.0 \mathrm{~mm}$ and $5.99 \mathrm{~mm}$, with $45.5 \%$ and $42.4 \%$ respectively. The present study shows the importance of integrating the septal papillary muscle to the International Anatomical Nomenclature.

KEY WORDS: Anatomy; Heart; Right ventricle; Septal papillary muscle.

\section{REFERENCIAS BIBLIOGRAFICAS}

Bouchet, A . \& Cuilleret, J. Anatomía descriptiva, topográfica y funcional. Buenos Aires, Médica Panamericana, 1979.

Drake, R.; Vogl, W. \& Mitchell, A. Anatomía para estudiantes. Madrid, Elsevier, 2005.

García-Porrero, J. \& Hurlé, J. Anatomía Humana. Madrid, McGraw-Hill Interamericana, 2005.

Gardner, E.; Gray, D. \& O'rahilly, R. Anatomía. 2ª edn. Barcelona, Salvat, 1971.

Goss, C. M. Gray Anatomía. Barcelona, Salvat, 1976.

Nigri, G. R.; Di Dio, L. J. \& Baptista, C. A. Papillary muscles and tendinous coros of the right ventricle of the human heart: morphological characteristics. Surg. Radiol. Anat., 23(1):45-9, 2001.

Quiroz, F. Tratado de Anatomía Humana. 32 Edn. México D. F., Porrúa, 1993. Vol. II.

Restivo, A.; Smith, A.; Wilkinson, J. \& Anderson, R. The medial papillary muscle complex and its related septomarginal trabeculation. A normal anatomical study on human hearts. J. Anat., 163:231-42, 1989.

Rouvière, H. \& Delmas, A. Anatomía Humana, Descriptiva, Topográfica y Funcional. 10 a Edn. París, Masson, 1999. Vol. II.

Skwarek, M.; Dudziak, M.; Hreczecha, J. \& Grzybiak, M.
The connection between the papillary muscles and leaflets of the tricuspid valve. Folia Morphol., 65(4):3228, 2006.

Snell, R. Anatomía Clínica. 6a Edn. México D. F., McGrawHill Interamericana, 2000.

Testut, L. \& Latarjet, A. Tratado de Anatomía Humana. 9a Edn. Barcelona, Salvat, 1972. Vol. II.

Wenink, A. C. The medial papillary complex. Br. Heart J., 39(9):1012-8, 1977.

Williams, P. \& Warwick, R. Anatomía. 36ª Edn. Barcelona, Salvat, 1985. Vol. I.

Dirección para correspondencia:

Aglae Soto Peralta

Universidad Católica del Maule

Talca

CHILE

Email: asoto@ucm.cl

Recibido : 09-05-2011

Aceptado: 16-07-2011 